\title{
Genetic Algorithm for Double Digest Problem
}

\author{
S. Sur-Kolay ${ }^{1}$, S. Banerjee ${ }^{2}$, S. Mukhopadhyaya ${ }^{1, \star}$, and C.A. Murthy ${ }^{1}$ \\ 1 Indian Statistical Institute, Kolkata, India \\ ${ }^{2}$ Honeywell Technology Solutions Lab Pvt. Ltd., Bangalore, India
}

\begin{abstract}
The strongly NP-complete Double Digest Problem (DDP) for physical mapping of DNA, is now used for efficient genotyping. An instance of DDP has multiple distinct solutions. Existing methods produce a single solution, and are slow for large instances. We employ a type of equivalence among the distinct solutions to obtain almost all of them. Our method comprises of first finding a solution from each equivalence class by an elitist genetic algorithm, and then generating entire classes. Notable efficiency was achieved due to significant reduction in search space.
\end{abstract}

\section{Introduction}

A DNA molecule can be represented as a string over the alphabet $\{A, C, G, T\}$ with its length in kilobasepairs, ranging from a few thousands for a yeast chromosome to billions for the entire human genome comprising of 23 pairs of chromosomes. A restriction enzyme can digest a DNA molecule and cut it at every occurrence of a short specific pattern. For example, the restriction enzyme PstI cuts each occurence of the string $C T G C A G$ in a DNA into two substrings $C T G C A$ and $G$. These locations are called cut sites of a DNA formed by the restriction enzyme. The sequence of cut sites constitute the restriction map of DNA [8], and the fragment between two consecutive cut sites is a restriction fragment for that enzyme. The lengths (but not the order!) of these fragments can be measured by gel-electrophoresis. In the Double Digest Problem (DDP), a restriction map is to be constructed from the fragment lengths obtained by applying two restriction enzymes separately as well as together.

Since the first successful restriction site mapping was achieved in 1970s [11, DDP has been studied intensely. It has been shown to be NP-complete [5] and then, strongly NP-complete [2]. Different heuristics such as integer linear programming, simulated annealing (SA), have been proposed [6]12|9]. Although SA was the best known method for solving DDP, its main drawbacks are that it produces only one solution and is fairly slow. A variation of DDP called enhanced double digest (EDD) problem [7, though NP-hard, can be solved in linear time only under certain constraints.

Finding all possible distinct solutions for a given instance, may be used for verifying the correctness of the sequence produced by fragment assembly. Though

\footnotetext{
* Partially supported by a grant from Dept. of Science and Technology, Govt. of India.
} 


\begin{tabular}{|c|c|c|c|}
\hline$A$ & 2 & 5 & 3 \\
\hline $\mathrm{B}$ & 3 & 3 & 4 \\
\hline & 2 & 3 & 3 \\
\hline
\end{tabular}

(a)

\begin{tabular}{|c|c|c|c|}
\hline 3 & 2 & & 5 \\
\hline 3 & 4 & & 3 \\
\hline 3 & 2 & 2 & 3 \\
\hline
\end{tabular}

(b)

Fig. 1. Two distinct orderings of the fragment lengths for enzymes $A$ and $B$

the number of solutions to DDP increases exponentially with the number of cut-sites, the entire set of solutions can be partitioned into a small number of equivalence classes [129].

In this paper, we design an elitist Genetic Algorithm to obtain all distinct non-equivalent solutions to DDP, from which the complete set of solutions is generated by applying simple transformations. The vanilla flavour genetic algorithm typically terminates with only one optimal solution even if there exist more than one. Hence, the proposed GA to produce all optimal solutions for DDP, which may be exponential in number of fragment lengths, is by itself novel.

\section{Problem Formulation}

The combinatorial description of Double Digest Problem (DDP) is as follows [12]: let $\mathcal{A}=\left\{a_{i}: 1 \leq i \leq m\right\}, \mathcal{B}=\left\{b_{i}: 1 \leq i \leq n\right\}$ and $\mathcal{A}+\mathcal{B}=\left\{d_{i}: 1 \leq i \leq l\right\}$ be the multisets of fragment lengths in non-decreasing order, obtained by application of restriction enzyme $A, B$, and $A+B$ ( $A$ and $B$ together) respectively on a DNA string of length $L$. Thus, $\sum_{i=1}^{m} a_{i}=\sum_{i=1}^{n} b_{i}=\sum_{i=1}^{l} d_{i}=L$.

Let $\sigma$ and $\mu$ be two permutations of the indices $(1,2, \ldots, m)$ and $(1,2, \ldots, n)$ respectively. In the restriction map $M$, the elements of $\mathcal{A}$ and $\mathcal{B}$ in their respective map orders $\sigma$ and $\mu$, are $A_{\sigma}=\left\langle A_{1}, \cdots, A_{m}\right\rangle$ and $B_{\mu}=\left\langle B_{1}, \cdots, B_{n}\right\rangle$ such that for $i<j, A_{i}\left(B_{i}\right)$ appears to the left of $A_{j}\left(B_{j}\right)$. While superposition of the sequences $A_{\sigma}$ and $B_{\mu}$ yields the sequence of fragment lengths $C_{\sigma, \mu}=<$ $C_{1}, \cdots, C_{l}>$, we term the multiset of fragment lengths in non-decreasing order $C(\sigma, \mu)=\left\{c_{1}, \cdots, c_{p}\right\}$ as the configuration $(\sigma, \mu)$. If a cut site in the superposition also occurs in both $\sigma$ and $\mu$, it is called coincident cut site (ccs). DDP is to find possible configurations $(\sigma, \mu)$ such that $C(\sigma, \mu)=\mathcal{A}+\mathcal{B}$.

Example 1: Given $\mathcal{A}=\{2,3,5\}, \mathcal{B}=\{3,3,4\}$ and $\mathcal{A}+\mathcal{B}=\{1,1,2,3,3\}$, the first $(\sigma, \mu)$ in Fig. $1(\mathrm{a})$ yields $C(\sigma, \mu)=\{1,1,2,3,3\}$, whereas the second one in Fig. 1(b) gives $\{2,2,3,3\}$. While the first matches $(\mathcal{A}+\mathcal{B})$, and hence corresponds to a correct restriction map, the second one is not a valid solution.

\subsection{Classifying Solutions of DDP}

The notion of equivalence has been defined over the large solution space of DDP in more than one ways [12. Equivalence classes help in finding all solutions efficiently. Checking equivalence of two maps is non-trivial. Of the known types of equivalence, cassette equivalence is suitable for computation. 


\subsection{Cassette Equivalence}

In a map $M$, any subsequence $I_{A}\left(I_{B}\right)$ of $A_{\sigma}\left(B_{\mu}\right)$ is called a block. For $A_{i} \in A_{\sigma}$ $\left(B_{i} \in B_{\mu}\right)$, each fragment in $B_{\mu}\left(A_{\sigma}\right)$, properly contained in $A_{i}\left(B_{i}\right)$ is called a non-border block of $A_{i}\left(B_{i}\right)$. For each pair $i, j$ with $1 \leq i \leq j \leq l$, let $I_{C}$ be the set of fragments from $C_{i}$ to $C_{j}$. An extended definition of cassette for $I_{C}$ is introduced for completeness' sake; earlier definition [9] assumed that coincident cut sites are absent. Two cases need to be considered:

Case 1: Neither of the boundaries of $I_{C}$ is a coincident cut site $(c c s)$.

A cassette defined by $I_{C}$ is the pair of sets of intervals $\left(I_{A}, I_{B}\right)$, where $I_{A}$ and $I_{B}$ are the sets of all blocks of $A$ and $B$ respectively that contain a block of $I_{C}$. In Fig. 2(b), the unique cassette defined by $I_{C}=\left\{C_{4}, C_{5}\right\}$ is shown.

Case 2: At least one of the boundaries of $I_{C}$ is a $c c s s_{i}$, excepting either boundary of the DNA.

In this case, additional cassettes are also defined by $I_{C}$ as follows. For a given $I_{C}$, we denote the predecessor of the left boundary of $I_{C}$ and the successor of its right boundary by $s_{l}$ and $s_{r}$ respectively. If the $c c s s_{i}$ is the left (right) boundary of $I_{C}$, then the interval $\left(s_{l}, s_{i}\right)\left(\left(s_{i}, s_{r}\right)\right)$ arising from either $A_{\sigma}$ or $B_{\mu}$ may also be included in $I_{A}$ or $I_{B}$ respectively to form an additional cassette for this $I_{C}$. Further, if $s_{l}\left(s_{r}\right)$ is also a $c c s$, then there are two additional cassettes depending on the inclusion of the interval $\left(s_{l}, s_{i}\right)\left(\left(s_{i}, s_{r}\right)\right)$ in either $I_{A}$ or $I_{B}$.

Result 1: For a given $I_{C}$, the total number of possible cassettes may be $2,3,4$, 6 , and 9 depending on whether its adjacent cut sites are $c c s$ 's.

Cassette Transformations: If two disjoint cassettes have their respective left and right overlaps equal [12, and each overlap comprises of only one double digest fragment $C_{i}$, then these two cassettes can be exchanged to form a new solution (Fig. 2(a)). If the two overlaps of a cassette have same absolute value but different signs, then the cassette can be reflected (Fig. 2(b).

Definition: $\left[A_{\sigma}, B_{\mu}\right]$ and $\left[A_{\sigma}^{\prime}, B_{\mu}^{\prime}\right]$ are cassette equivalent if and only if there is a sequence of cassette transformations and permutations of the non-border block uncut fragments transforming $\left[A_{\sigma}, B_{\mu}\right]$ into $\left[A_{\sigma}^{\prime}, B_{\mu}^{\prime}\right]$.

Suppose $X$ is a subsequence of $A_{\sigma}$ or $B_{\mu}$. Let $I(X)=\left\{C_{i}: C_{i} \subseteq X\right\}$, $I_{s}(X)=\left\{\left|C_{i}\right|: C_{i} \subseteq X\right\}$ and $I_{s}^{*}(X)=\left\{\left|C_{i}\right|: C_{i}\right.$ is a border block of $\left.X\right\}$ if $|I(X)|>1$. The characteristic of a cassette equivalence class is defined as $I^{*} D$ $=\left(\left\{\left(I_{s}^{*}\left(A_{i}\right), I_{s}\left(A_{i}\right)\right): A_{i} \in A\right\},\left\{\left(I_{s}^{*}\left(B_{j}\right), I_{s}\left(B_{j}\right)\right): B_{j} \in B\right\}\right)$.

(a)

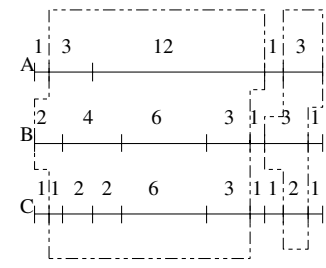

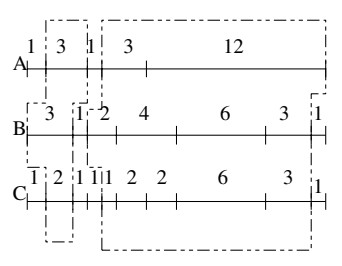

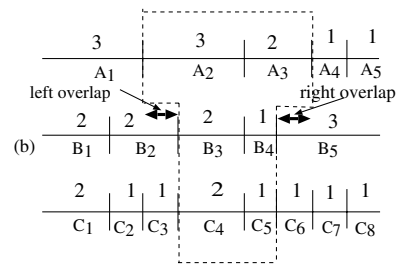

Fig. 2. Cassette transformations (a) exchange, (b) reflection 
Result $2[\mathbf{9}]:\left[A_{\sigma}, B_{\mu}\right]$ and $\left[A_{\sigma}^{\prime}, B_{\mu}^{\prime}\right]$ are cassette equivalent if and only if $I^{*} D$ $\left[A_{\sigma}, B_{\mu}\right]=I^{*} D\left[A_{\sigma}^{\prime}, B_{\mu}^{\prime}\right]$.

This result provides an efficient method for checking cassette equivalence. But it does not hold for a ccs if the definition of a cassette is restricted to Case 1 only. This motivated us to introduce a complete definition of cassette above, thereby enabling us to employ Result 2 in general.

\section{Proposed Algorithm for DDP}

Our proposed algorithm consists of two parts: first, an elitist genetic algorithm [4] to generate all possible valid non-equivalent (w.r.t. cassette equivalence) solutions to a given instance of DDP, and second an algorithm to generate all valid solutions from the set of representative solutions obtained in the first part, by performing simple cassette transformations in an efficient manner.

\subsection{GA for Finding Non-equivalent Solutions to DDP}

In order to allow for multiple optimum solutions in GA, the structure of classical GA needs to be modified in our proposed algorithm, as outlined below followed by details of string representation, cost function and modified genetic operators.

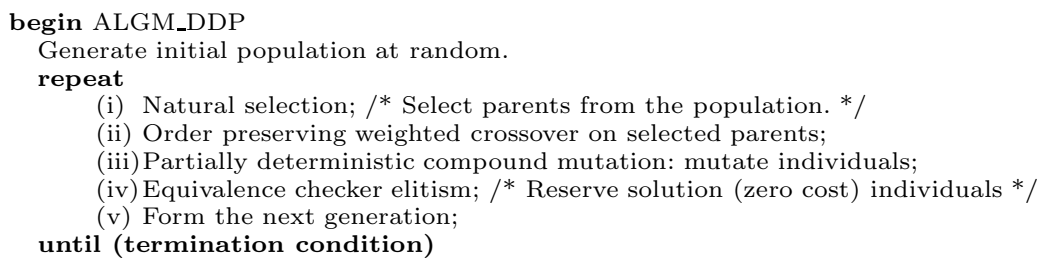

String Representation and Cost Function: A string is a configuration in DDP represented as a 2-tuple $(\sigma, \mu)$. Its cost is defined as $\zeta=\sum_{i=1}^{\max (p, l)}\left(d_{i}-c_{i}\right)^{2}$. If $p \neq l$, the shorter sequence is padded with zeroes. The optimum value of $\zeta$ is 0 which occurs if and only if the configuration is a valid solution.

Genetic Operators: Of the four genetic operations natural selection, crossover, mutation and elitism, which are applied stochastically, all operations save natural selection done by traditional roulette wheel, are modified to suit DDP.

Order Preserving Weighted Crossover: The single-point crossover on two configurations, where either the $A_{\sigma}$ s or the $B_{\mu}$ s are chosen as parents, is demonstrated below.

Example 2: Suppose the randomly chosen crossover point is $3, p_{1}=(1,3,2,1,3,4,2,2)$ and $p_{2}=(1,2,2,2,4,3,3,1)$. The child $c_{1}\left(c_{2}\right)$ is a permutation of $p_{1}\left(p_{2}\right)$ but inherits the order of the elements to the left of the crossover point from $p_{1}\left(p_{2}\right)$, and to the right from $p_{2}\left(p_{1}\right)$. Thus, $c_{1}=(1,3,2,2,2,4,3,1)$ and $c_{2}=(1,2,2,3,1,3,4,2)$.

Remark: The above crossover cannot generate any invalid permutation and can explore the search space efficiently. 
Partially Deterministic Compound Mutation: Given a string to be mutated, either $A_{\sigma}$ or $B_{\mu}$ is chosen randomly, say $A_{\sigma}$. All its elements are mutated by choosing a random number $r \in[0,1]$; if $r$ is less than the mutation probability then for each $i, 1 \leq i \leq m$, another random integer $j, 1 \leq j \leq m$, is generated, and $A_{i}$ and $A_{j}$ are swapped. This guarantees that a string can be mutated to any other string in the search space in one step. This condition ensures uniform convergence of the elitist model of GA to optimal solution.

Equivalence Checker Elitism: This preserves best strings across generations in a special array, subject to checking cassette equivalence with all other existing non-equivalent solution strings in the array. For equivalence checking, Result 2 of Sec. 2.2 is used. The next generation is formed from the union of the previous and present generations by including all the valid solutions, along with randomly chosen non-zero cost strings, keeping the population size invariant.

With this set of four genetic operations, the probability that all non equivalent strings are stored in the special array after sufficient number of iterations is almost 1 .

\subsection{Generating a Cassette Equivalence Class from a Representative}

From a representative string $s$ reserved in the special array above, GEN_CLASS $(s)$ produces the set E-CLASS of all strings equivalent to $s$ by calling PERMUT and CASS_EX_RF respectively for permutations of uncut fragments of non-border blocks and cassette transformations of $s$.

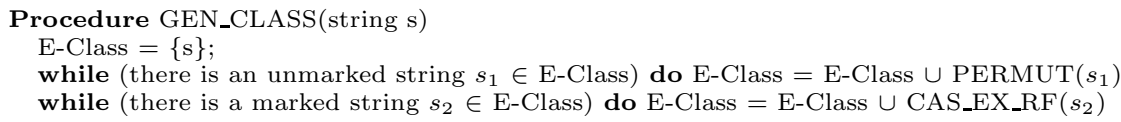

Given a map $M$, not all the $O\left(l^{2}\right)$ possible cassettes where $l$ is the number of cut sites in $(\mathcal{A}+\mathcal{B})$, are eligible for cassette transformations. We introduce a data structure called cassette generation table $(C G T)$ to operate on only eligible cassettes. The $i^{t h}$ row corresponds to cut site $i$ and contains its distance from the left boundary of the DNA, name of enzyme(s) responsible for it, left and right overlaps if it is respectively the left and right boundary of a cassette. To reduce search, entries with equal left (right) overlaps form a singly linked list.

Complexity of the Proposed Approach: The time complexity for the GA is $O\left(N k l^{2} \rho\right)$, where $l, \rho, N$ and $k$ are respectively cardinality of $\mathcal{A}+\mathcal{B}$, number of equivalence classes, size of the population and number of generations. The second phase requires $O\left(l^{3} \cdot T\right)$ time for $T$ distinct solutions. The space complexity of the GA is $O((N+\rho) l)$, and is output sensitive in second phase.

\section{Experimental Results and Discussion}

The ALGM_DDP was implemented in C, tested on DDP instances from laboratory data and artificial ones with fragment lengths rounded off (w.l.o.g.) to 
Table 1. Results of proposed method

\begin{tabular}{|c|c|c|c|c|c|}
\hline Problem Instance & \begin{tabular}{|c|} 
\# Equiv. \\
Classes
\end{tabular} & $\begin{array}{c}\# \\
\text { soln. }\end{array}$ & Problem Instance & $\begin{array}{c}\text { \# Equiv. } \\
\text { Classes }\end{array}$ & $\begin{array}{c}\# \\
\text { soln. }\end{array}$ \\
\hline $\begin{array}{c}\mathcal{A}=\{1,2,3,3,4,4,5,5\} \\
\mathcal{B}=\{1,2,3,3,3,7,8\} \\
\mathcal{A}+\mathcal{B}=\{1,1,1,1,1,1,2,2,2,2,2,3,4,4\}\end{array}$ & 393 & 36660 & $\begin{aligned} \mathcal{A} & =\{1,2,2,3,3,4\} \\
\mathcal{B} & =\{1,1,2,2,4,5\} \\
\mathcal{A}+\mathcal{B} & =\{1,1,1,1,1,2,2,33\}\end{aligned}$ & 18 & 3210 \\
\hline $\begin{array}{c}\mathcal{A}=\{5509,5626,6527,6766,7233,16841\} \\
\mathcal{B}=\{3526,4878.5643,5804,7421,21230\} \\
\mathcal{A}+\mathcal{B}=\{1120,1868,2564,2752,3240, \\
3526,3758,3775,4669,5509,15721\}\end{array}$ & 1 & 2 & 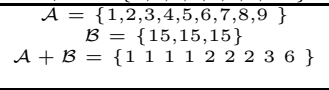 & 172 & 15840 \\
\hline $\begin{array}{c}\mathcal{A}=\{7,8,18\} \\
\mathcal{B}=\{1,2,2,4,5,5,5,9\} \\
\mathcal{A}+\mathcal{B}=\{1,2,2,2,2,3,4,5,5,7\}\end{array}$ & 52 & 686 & $\begin{array}{c}\mathcal{A}=\{7,8,18\} \\
\mathcal{B}=\{1,1,1,1,2,3,5,7,12\} \\
\mathcal{A}+\mathcal{B}=\{1,1,1,1,1,2,2,4,5,7,8\}\end{array}$ & 65 & 930 \\
\hline $\begin{array}{c}\mathcal{A}=\{1,1,1,1,2,3,5,7,12\} \\
\mathcal{B}=\{1,2,2,4,5,5,5,9\} \\
\mathcal{A}+\mathcal{B}=\{1,1,1,1,1,1,1,1,1,2,2,3,3,5,9\}\end{array}$ & 376 & 92920 & $\begin{array}{c}\mathcal{A}=\{1,3,3,12\} \\
\mathcal{B}=\{1,2,3,3,4,6\} \\
\mathcal{A}+\mathcal{B}=\{1,1,1,1,2,2,2,3,6\}\end{array}$ & 18 & 208 \\
\hline
\end{tabular}

integral values keeping the sum of the lengthd invariant. The results appear in Table 1 for eight such problems, three being laboratory data for D10 using (BamHI, PstI), (BamHI, HindIII) and (PstI, HindIII) respectively.

The values of $N$ and $k$ were 50 and 10,000. For each instance, 5 initial populations and for each initial population, 10 trials were performed. Crossover probability was 0.85 . Mutation probability was varied linearly in cycles of 200 iterations from $2 /(m+n)$ to 0.45 and back to $2 /(m+n)$, to enable efficient exploration of the search space and to prevent the GA from getting stuck in a local optima [1]. For all the instances, convergence occurred within the first 5,000 iterations. The number of distinct solutions was known a priori only for the last instance and it tallied with our results. For any instance, the proposed GA can theoretically produce all the distinct solutions after sufficiently large number of iterations [1].

Lastly, the efficacy of a search heuristic is judged by its reduction in search space. For Instances 1, 4 and 6 (in column-major order) of Table 1, the space reduction figures are $77 \%, 88 \%$ and $99 \%$ respectively.

In summary, our algorithm finds all valid solutions to a given instance of DDP efficently. For larger problem instances, the reduction in search space attained by the proposed method is remarkably high. It would be worth experimenting with the cost function as well as incorporate ideas of mulit-niche crowding [3]. Another future work is to tune the algorithm for either incompletely specified or inaccurate data.

\section{References}

1. Bhandari, D., Murthy, C. A., and Pal, S. K.: Genetic algorithm with elitist model and its convergence. International Journal of Pattern Recognition and Artificial Intelligence, 10, 1147 - 1151, 1999.

2. Cieliebak, M., Eidenbenz, S. and Woeginger, G. J.: Double digest revisited: Complexity and approximability in the presence of noisy data. Technical Report No. 382, ETH Zurich, Department of Computer Science, 2002.

3. Cedeno, W., Vemuri, V. Rao and Slezak, T.: Multi-niche crowding in genetic algorithms and its application to the assembly of DNA Restriction-fragments. Evolutionary Computation, 2(4), 321-345, 1994. 
4. Goldberg, D. E.: Genetic Algorithm in Search, Optimization and Machine Learning. Addison-Wesley, New York, 1989.

5. Goldstein, L. and Waterman, M. S.: Mapping DNA by stochastic relaxation. Advances in Applied Mathematics, 8, 194-207, 1987.

6. Inglehart, J. and Nelson, P. C.: On the limitations of automated restriction mapping. Computer Applications in the Biosciences (CABIOS), 10(3), 249 - 261, 1994.

7. Kao, M-Y, Samet, J. and Sung, W-K.: The enhanced double digest problem for DNA physical mapping. Proc. $7^{\text {th }}$ Scandinavian Workshop on Algorithm Theory (SWAT00), 383 - 392, 2000.

8. Nathans, D. and Smith, H. O.: Restriction endonucleases in the analysis and restructuring of DNA molecules. Annual Review of Biochemistry, 44, 273-293, 1975.

9. Pevzner, P. A.: Computational Molecular Biology: An Algorithmic Approach. The MIT Press, MA, 2000.

10. Schmitt, W. and Waterman, M. S.: Multiple solutions of DNA restriction mapping problem. Advances in Applied Mathematics, 12, 412-427, 1991.

11. Smith, H. O. and Wilcox, K. W.: A restriction enzyme from Hemophilus influenzae. I. Purification and general properties. Journal of Molecular Biology, 147, 379-391, 1970.

12. Waterman, M. S.: Introduction to Computational Biology : Maps, sequences and genomes. Chapman \& Hall, UK, 1995. 